\title{
Clostridium beijerinckii
}

National Cancer Institute

\section{Source}

National Cancer Institute. Clostridium beijerinckii. NCI Thesaurus. Code C86271.

A species of anaerobic, Gram positive, rod shaped bacteria assigned to the phylum

Proteobacteria. This species is motile, sporulating, sacchrolytic, mesophilic, and produces acetate, butanol, ethanol, acetoin and acetyl methyl carbonil from fermentation. C.

beijerinckii is found in the soil and feces and is an important organism used in commercial solvent production. 